\title{
AVNeo (Ozaki) and transaortic mitral valve repair using autologous pericardium only for aortomitral endocarditis
}

\author{
Umberto Benedetto, PhD, Szabolcs Gergely, Arnaldo Dimagli, MD, and \\ Shubhra Sinha, MBBS, Bristol, United Kingdom
}

\footnotetext{
From the Bristol Heart Institute, University of Bristol, Bristol, United Kingdom.

This study was funded by the Bristol Biomedical Research Centre (NIHR Bristol BRC).

Disclosures: The authors reported no conflicts of interest.

The Journal policy requires editors and reviewers to disclose conflicts of interest and to decline handling or reviewing manuscripts for which they may have a conflict of interest. The editors and reviewers of this article have no conflicts of interest.

Received for publication April 16, 2020; revisions received April 16, 2020; accepted for publication May 8, 2020; available ahead of print May 17, 2020.

Address for reprints: Umberto Benedetto, PhD, University of Bristol, Bristol Heart Institute, Bristol Royal Infirmary, Upper Maudlin St, BS2 8HW Bristol, United Kingdom (E-mail: Umberto.Benedetto@ Bristol.ac.uk). JTCVS Techniques 2020;3:101-3

2666-2507

Copyright (c) 2020 The Authors. Published by Elsevier Inc. on behalf of The American Association for Thoracic Surgery. This is an open access article under the CC BY-NC-ND license (http://creativecommons.org/licenses/bync-nd/4.0/).

https://doi.org/10.1016/j.xjtc.2020.05.006
}

Video clip is available online.

Aortomitral valve infective endocarditis (IE) is a very challenging clinical condition. Conventional surgery with multiple surgical incisions and prosthetic valve implantation remains associated with suboptimal outcomes and more than $50 \%$ of patients requiring reintervention. ${ }^{1}$ We present the first case of aortomitral valve IE treated with a total biological solution by combining the aortic valve neocuspidization (AVNeo; Ozaki procedure) ${ }^{2}$ and mitral valve repair with the exclusive use of autologous pericardium through a total transaortic approach. AVNeo with glutaraldehyde-treated autologous pericardium has been associated with excellent midterm results. Ozaki and colleagues $^{2}$ reported only 2 cases of structural valve degeneration out of 850 procedures after a maximum follow-up of 8 years.

Whereas mitral valve repair with autologous pericardium is a well-recognized strategy for patients with mitral $\mathrm{IE}^{3}$ AVNeo has been reported primarily in patients with degenerative aortic valve disease. ${ }^{2}$ The use of AVNeo in aortomitral valve IE represents a very attractive strategy, extending the use of autologous pericardium not only for mitral valve repair, but also for the treatment of the aortic valve. This strategy avoids any prosthetic material, thereby minimizing the risk of reinfection. We also adopted a transaortic approach for the treatment of anterior mitral valve leaflet perforation. This approach provides excellent exposure of

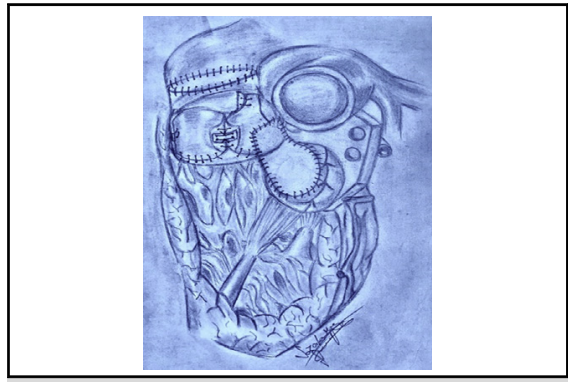

Anatomic drawing showing mitral valve patch and aortic valve neocuspidization with autologous pericardium to treat aortomitral endocarditis.

\section{CENTRAL MESSAGE \\ We present a case of aortomitral infective endocarditis treated with a totally biological solution by combining AVNeo (Ozaki procedure) and mitral valve repair using autologous pericar- dium through a total transaortic approach.}

See Commentaries on pages 104, 106, and 108

the ventricular aspect anterior mitral valve leaflet ${ }^{4}$ and minimizes surgical trauma by avoiding a left atriotomy. This reduces the risk of bleeding, which is particularly relevant in the presence of IE-induced coagulopathy. ${ }^{5}$

\section{CASE DESCRIPTION}

A 50-year-old man was admitted with malaise, pyrexia, and a new murmur but no other preexisting comorbidities. Transesophageal echocardiography (TEE) showed trileaflet AV endocarditis with severe aortic valve insufficiency and perforation of the anterior mitral valve leaflet with severe mitral regurgitation and the decision for emergency surgery was made. His preoperative EuroSCORE II was $2.41 \%$. We routinely perform AVNeo in patients with aortic valve diseases, including aortic valve IE, and this technique was chosen in the present case in combination with anterior mitral leaflet repair. After full sternotomy, autologous pericardium 


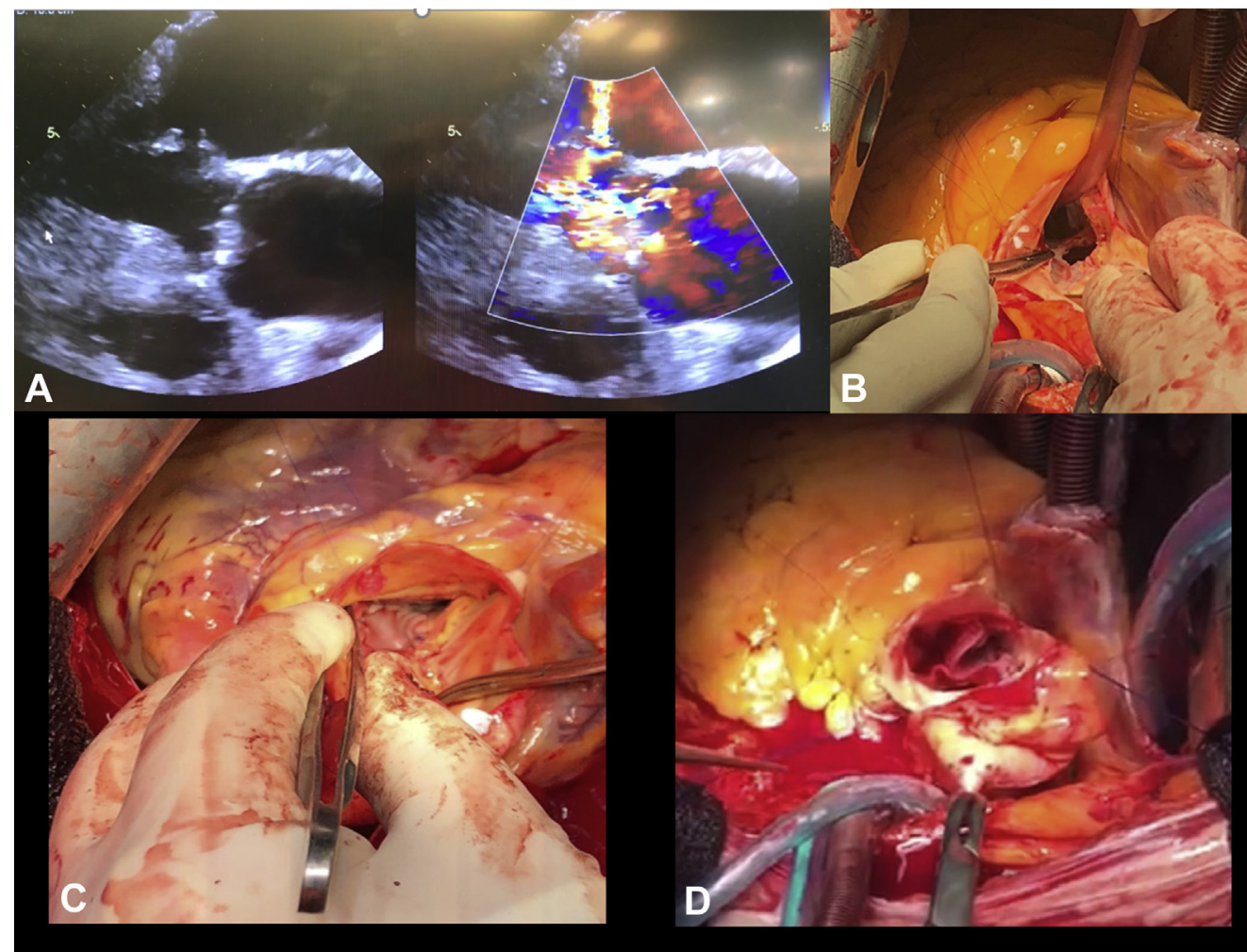

FIGURE 1. A, Transesophageal echocardiography showing aortomitral endocarditis with severe mitral valve regurgitation. B, Transaortic approach for anterior mitral valve excision. C, Transaortic approach for anterior mitral valve patch with autologous pericardium. D, Aortic valve neocuspidization using autologous pericardium.

was harvested between the 2 phrenic nerves and treated with glutaraldehyde and saline according to protocol. ${ }^{3}$ After systemic heparinization, cardiopulmonary bypass (CPB) was instituted via standard cannulation, and anterograde cold blood was delivered directly to the ostia for cardioplegic arrest.

The surgical view confirmed acute endocarditis with extensive destruction of all 3 aortic valve leaflets and anterior mitral valve leaflet perforation. The aortic valve leaflets were excised. The aortotomy was extended into the noncoronary aortic sinus to provide good exposure of the anterior mitral valve leaflet, and two 5-0 Prolene sutures were placed through healthy mitral valve chords and used to lift it up. The anterior mitral valve leaflet was excised almost entirely except for the edge of the anterior mitral leaflet where marginal chordae (primary chordae) were inserted. A patch of autologous pericardium was trimmed to size and used to repair the anterior mitral valve leaflet with $4 / 0$ Prolene running suture. The superior edge of the mitral patch was extended into the noncoronary sinus to reinforce the mitro-aorto continuity.

AVNeo with autologous pericardium was performed using 3 size 31 leaflets (Figures 1 and 2) implanted with three $4 / 0$ Prolene running sutures. The patient was successfully weaned from $\mathrm{CPB}$, and TEE showed no significant aortic

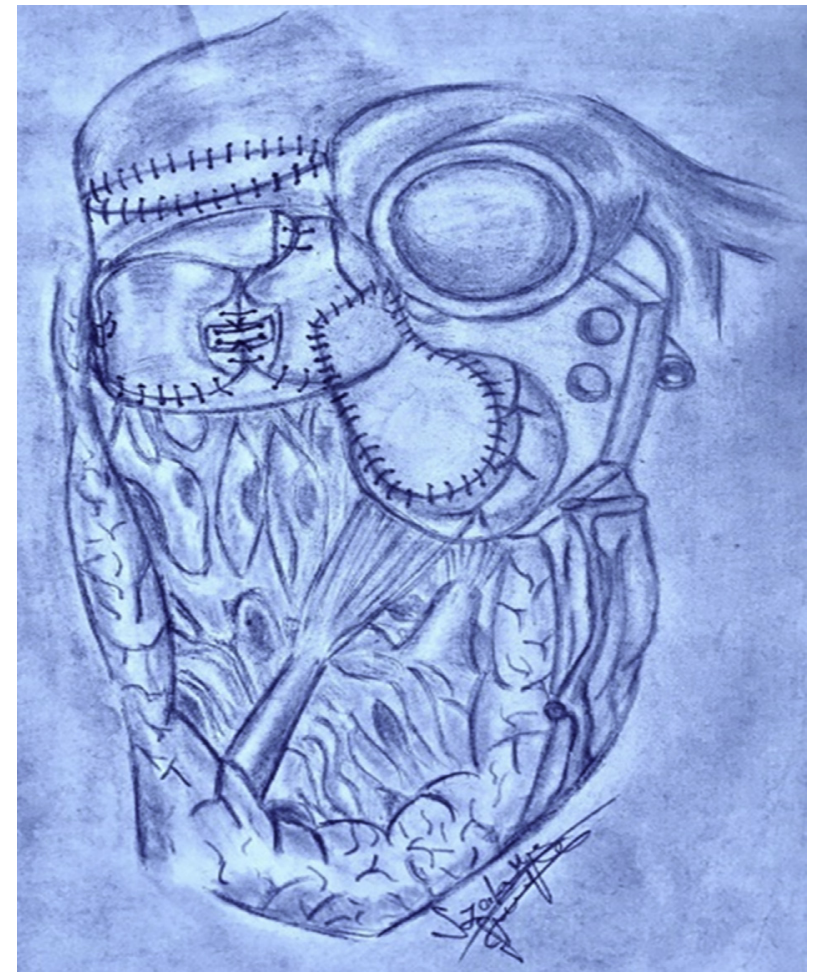

FIGURE 2. Anatomic drawing showing mitral valve patch and aortic valve neocuspidization with autologous pericardium to treat aortomitral endocarditis. 


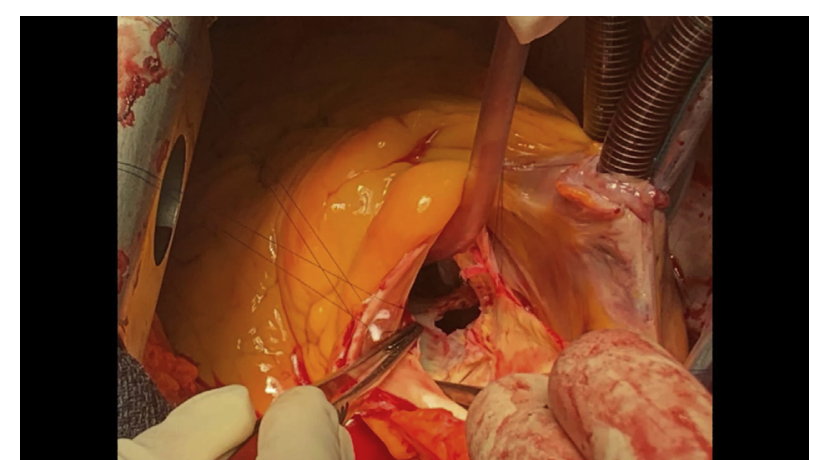

VIDEO 1. Preoperative transesophageal echocardiography (TEE) showing aortomitral infective endocarditis, performance of the surgical procedure, and 6-month follow-up TEE confirming well-functioning mitral and aortic valves. Video available at: https://www.jtcvs.org/article/S26662507(20)30227-3/fulltext.

or mitral regurgitation and low gradients across both valves. The total aortic cross-clamp and CPB times were 89 minutes and 100 minutes, respectively. Long-term acetylsalicylic acid (ASA) $75 \mathrm{mg}$ daily was administrated postoperatively.
Streptococcus sanguinis was isolated by blood cultures and intraoperative specimens, and targeted antimicrobial therapy (benzylpenicillin) was administered for 6 weeks, with complete resolution of the infection.

After discharge, a 6-month follow-up TEE confirmed well-functioning mitral and aortic valves with no evidence of recurrent infection or valve thrombosis (Video 1). The patient provided informed consent for publication of the study data.

\section{References}

1. Navia JL, Elgharably H, Hakim AH, Witten JC, Haupt MJ, Germano E, et al. Long-term outcomes of surgery for invasive valvular endocarditis involving the aortomitral fibrosa. Ann Thorac Surg. 2019;108:1314-23.

2. Ozaki S, Kawase I, Yamashita H, Uchida S, Takatoh M, Kiyohara N. Midterm outcomes after aortic valve neocuspidization with glutaraldehyde-treated autologous pericardium. J Thorac Cardiovasc Surg. 2018;155:2379-87.

3. Quinn RW, Wang L, Foster N, Pasrija C, Ghoreishi M, Dawood M, et al. Longterm performance of fresh autologous pericardium for mitral valve leaflet repair. Ann Thorac Surg. 2020;109:36-41.

4. Hosseini MT, Chandrasekaran V. Transaortic mitral valve repair in double valve infective endocarditis. J Card Surg. 2010;25:651-3.

5. Buyukasýk NS, Ileri M, Alper A, Senen K, Atak R, Hisar I, et al. Increased blood coagulation and platelet activation in patients with infective endocarditis and embolic events. Clin Cardiol. 2004;27:154-8. 